\title{
Caffeine ameliorates hyperoxia-induced lung injury by protecting GCH1 function in neonatal rat pups
}

\author{
Xigang Jing ${ }^{1}$, Yi-Wen Huang ${ }^{2}$, Jason Jarzembowski ${ }^{3}$, Yang Shi ${ }^{4}$, Girija G. Konduri ${ }^{1}$ and Ru-Jeng Teng ${ }^{1}$
}

BACKGROUND: Bronchopulmonary dysplasia (BPD) is a major morbidity in premature infants, and impaired angiogenesis is considered a major contributor to BPD. Early caffeine treatment decreases the incidence of BPD; the mechanism remains incompletely understood.

METHODS: Sprague-Dawley rat pups exposed to normoxia or hyperoxia since birth were treated daily with either $20 \mathrm{mg} / \mathrm{kg}$ caffeine or normal saline by an intraperitoneal injection from day 2 of life. The lungs were obtained for studies at days 10 and 21. RESULTS: Hyperoxia impaired somatic growth and lung growth in the rat pups. The impaired lung growth during hyperoxia was associated with decreased levels of cyclic AMP (CAMP) and tetrahydrobiopterin (BH4) in the lungs. Early caffeine treatment increased CAMP levels in the lungs of hyperoxia-exposed pups. Caffeine also increased the levels of phosphorylated endothelial nitric oxide synthase (eNOS) at serine ${ }^{1177}$, total and serine ${ }^{51}$ phosphorylated GTP cyclohydrolase $1(\mathrm{GCH} 1)$, and $\mathrm{BH} 4$ levels, with improved alveolar structure and angiogenesis in hyperoxia-exposed lungs. Reduced GCH1 levels in hyperoxia were due, in part, to increased degradation by the ubiquitin-proteasome system.

CONCLUSION: Our data support the notion that early caffeine treatment can protect immature lungs from hyperoxia-induced damage by improving eNOS activity through increased $\mathrm{BH} 4$ bioavailability.

$\mathbf{P}$ reterm infants are born with immature lungs and frequently require oxygen support and mechanical ventilation. They are at a high risk for lung injury, which can progress to chronic lung disease or bronchopulmonary dysplasia (BPD). BPD represents an arrest of lung development, initiated by inflammation and oxidative stress $(1,2)$ Impaired angiogenesis is considered a key contributor to BPD (3).

Both supplemental oxygen (4) and mechanical ventilation (5) increase reactive oxygen species formation and deplete nitric oxide (NO) availability in the lungs. Decreased formation of NO by endothelial nitric oxide synthase (eNOS) in pulmonary vascular endothelial cells contributes to impaired angiogenesis (6). Thus, maintaining adequate endogenous
eNOS activity may prevent preterm infants from developing BPD.

Adequate eNOS coupling requires the interaction of eNOS with multiple cofactors. Tetrahydrobiopterin (BH4) is an essential cofactor for all members of the NOS family $(7,8)$. BH4 deficiency uncouples eNOS, which leads to superoxide formation instead of NO formation, when NOS is activated. Uncoupled eNOS has been shown to be the major source of oxidative stress in a rat model of ventilator-induced lung injury, which was improved by $\mathrm{BH} 4$ supplementation (9).

Rat pups are born at the saccular stage of lung development, similar to premature infants at 24-28 weeks' gestation, and they reach the alveolar stage between postnatal days 4 (P4) and 14 (P14) (10). The histological changes that develop after exposure to hyperoxia in rat pups mimic BPD in premature human infants (11). Caffeine, a nonselective phosphodiesterase inhibitor, is commonly used to treat apnea of prematurity $(12,13)$. The benefit of early caffeine treatment in preventing BPD had not been recognized until a randomized clinical trial identified this unexpected association in the secondary analysis of data (14). A recent retrospective study by Canadian researchers further supported the efficacy of caffeine in preventing BPD (15). However, the mechanisms involved in the lung protective effect of caffeine remain unclear.

It has been previously shown that cyclic AMP (cAMP) increases GTP cyclohydrolase 1 (GCH1) protein levels in endothelial cells (16), and phosphorylation enhances GCH1 activity (17). GCH1 is the rate-limiting enzyme for $\mathrm{BH} 4$ synthesis in endothelial cells. We previously reported that $\mathrm{BH} 4$ supplementation in the presence of oxidative stress recouples eNOS and improves in vitro angiogenesis (18). We hypothesized that hyperoxia decreases GCH1 levels and activity in the developing lungs, whereas early caffeine treatment protects the developing lungs from hyperoxia-induced injury through increased cAMP levels and improved GCH1 function, which leads to eNOS recoupling. Our results show that hyperoxia decreases cAMP and BH4 levels in the lungs, whereas early caffeine treatment protects the developing lungs from hyperoxic injury by increasing $\mathrm{BH} 4$ levels. Our findings provide the

\footnotetext{
${ }^{1}$ Department of Pediatrics, Medical College of Wisconsin, Milwaukee, Wisconsin; ${ }^{2}$ Department of Obstetrics and Gynecology, Medical College of Wisconsin, Milwaukee, Wisconsin; ${ }^{3}$ Department of Pathology, Medical College of Wisconsin, Milwaukee, Wisconsin; ${ }^{4}$ Patient Centered Research, Aurora Health Care, Milwaukee, Wisconsin. Correspondence: Ru-Jeng Teng (rteng@mcw.edu)

Received 1 December 2016; accepted 21 March 2017; advance online publication 24 May 2017. doi:10.1038/pr.2017.89
} 
biological basis for the role of early caffeine treatment in the prevention of BPD in premature infants.

\section{METHODS}

\section{Antibodies and Chemicals}

Rabbit anti-phospho-eNOS serine ${ }^{1177}$ (p-eNOS $^{1177}$ ) antibody was from Cell Signaling (Beverly, MA); mouse anti-GCH1 antibody was from Abnova (Taipei, Taiwan); rabbit anti-DHFR antibody was from GeneTex (Irvine, TX); mouse anti- $\beta$-actin antibody was from SigmaAldrich (St Louis, MO); rabbit anti-VEGF antibody was from Santa Cruz (Dallas, TX); mouse anti-ubiquitin antibody was from Thermo Fisher Scientific (Waltham, MA); rabbit anti-rat endothelial cell antigen antibody was from Abcam (Cambridge, MA); horse raddish peroxidase-tagged secondary antibodies for western blot were from Bio-Rad (Hercules, CA); biotinylated horse anti-rabbit IgG and horse anti-mouse IgG were from Vector Laboratories (Burlingame, CA). Rabbit anti-phospho-GCH1 serine ${ }^{51}$ was custom-made by 21 st Century Biochemicals (Marlborough, MA, USA) using a keyhole limpet hemocyanin-conjugated short peptide based on rat GCH1 sequence.

The Caffeine/Pentoxifylline ELISA kit was from Neogen (Lexinton, KY); the direct cAMP enzyme immunoassay kit was from Arbor Assays (Ann Arbor, MI); the EZ DNA Methylation kit was from Zymo Research (Irvine, CA); PyroMark Gold Q96 Reagents for performing pyrosequencing reactions were from Qiagen (Germantown, MD); the PureLink genomic DNA mini kit was from Invitrogen (Carlsbad, CA); Van Giesons and Resorcin-Fuchsin solutions were from Electron Microscopy Sciences (Hatfield, PA); the bicinchoninic acid (BCA) protein assay kit was from Thermo Scientific (Rockford, IL); Vectastain ABC kit and Bloxall blocking solution were from Vector Laboratories; protein block solution, antibody diluent, and liquid diaminobenzidine (DAB)+substrate chromogen system were from Dako (Carpinteria, CA); the In situ cell death detection kit was from Roche (Indianapolis, IN); all other chemicals were from Sigma-Aldrich.

\section{Animal Care}

Animal use was approved by the Institutional Animal Care and Use Committee of Medical College of Wisconsin (AUA\#2268; Figure 1). Rats were cared under 12-h day/night cycle with unlimited access to chow and water. For each experiment $(n=5)$, four litters of rat pups were exposed simultaneously, within $12 \mathrm{~h}$ of birth, to either hyperoxia ( $>90 \%$ oxygen chamber) or normoxia (room air) until day 10 of life (P10). Oxygen concentrations were monitored continuously with an oxygen sensor (Drägerwerk AG, Lübeck, Germany). Within each assigned group, pups received either daily intraperitoneal injections of $20 \mathrm{mg} / \mathrm{kg}$ caffeine base or the same volume of normal saline as a control from P1 to P21. Nursing dams were rotated between normoxia and hyperoxia litter every day to reduce $\mathrm{O}_{2}$ toxicity. All remaining pups were recovered in room air from P11 to P21. The lungs from at least six pups per group were obtained at P10 and P21 for histology and downstream assays.

\section{Body Weight}

Rat pups were checked and weighed at 0900 hours every day by an electronic scale to the closest gram.

\section{Determination of Serum Caffeine Levels}

Blood was collected at the time of killing. To obtain serum, the whole blood samples were centrifuged at $2,000 \mathrm{~g}$ for $10 \mathrm{~min}$ at $4^{\circ} \mathrm{C}$ after they were clotted. Serum caffeine levels were determined at P10 and P21 using the Caffeine/Pentoxifylline ELISA test kit according to the manufacturer's protocol. Standards were freshly prepared prior to the assay.

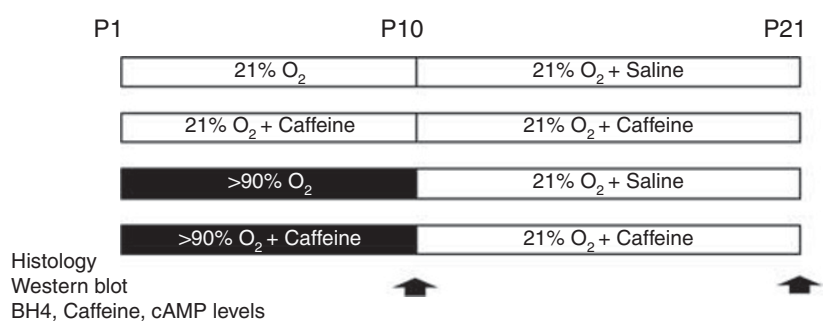

Figure 1. Study protocol for exposure of pups to hyperoxia and caffeine. $\mathrm{P} 1, \mathrm{P} 10$, and $\mathrm{P} 21$ are postnatal days 1,10 , and 21 , respectively.

\section{Measurement CAMP Levels}

Aliquots of lung homogenates were assayed for the levels of cAMP using the direct cAMP enzyme immunoassay kit and were expressed as $\mathrm{pmol} / \mathrm{mg}$-protein.

\section{Western Blotting}

Lung tissue was homogenized in MOPS buffer $(20 \mathrm{mM} 3-\mathrm{N}$ morpholino-propanesulfonic acid, $2 \mathrm{mM}$ EGTA, $5 \mathrm{mM}$ EDTA, $30 \mathrm{mM} \mathrm{NaF}, 10 \mathrm{mM} \beta$-glycerophosphate, $10 \mathrm{mM}$ Na pyrophosphate, $2 \mathrm{mM} \mathrm{Na}$ orthovanadate, $1 \mathrm{mM}$ PMSF, $0.5 \% \mathrm{NP}-40,1 \%$ protease inhibitor cocktail, and 1\% phosphatase inhibitor cocktails 2 and 3, $\mathrm{pH}$ 7.0) using the Bullet Blender (Next Advance, Averill Park, NY) to obtain tissue homogenate. For western blotting analysis, $30 \mu \mathrm{g}$ of lysate protein was resolved by SDS-polyacrylamide gel electrophoresis, transferred to nitrocellulose membranes $(0.2 \mu \mathrm{m})$, and then probed with VEGF, eNOS, p-eNOS ${ }^{1177}, \mathrm{GCH} 1, \mathrm{p}-\mathrm{GCH} 1^{51}$, and DHFR antibodies overnight at $4{ }^{\circ} \mathrm{C}$. Signals were generated after incubation with horseradish peroxidase-conjugated goat anti-rabbit $(1: 10,000)$ or anti-mouse $(1: 10,000)$ IgG using the SuperSignal West Pico (Pierce, Waltham, MA), or Femto mixed with Pico for some low abundant proteins, and recorded on CL-Xposure films. Integrated optical density was processed using Image J, and the $\beta$-actin signal was used as a loading control.

\section{Quantification of $\mathrm{BH} 4$}

$\mathrm{BH} 4$ was assayed by the high-performance liquid chromatography method using an electrochemical detector (ESA Biosciences CoulArray System, Chelmsford, MA), as previously described (19). Multichannel coulometric detection was set between 0 and $600 \mathrm{mV}$. Intracellular concentrations were calculated using authentic $\mathrm{BH} 4$ standards. BH4 levels were then normalized to protein concentrations.

\section{Histology}

For histology studies, the trachea was cannulated by an Instech Solomon (20 G) stainless steel feeding tube (Plymouth Meeting, PA), and the lungs were inflated with $10 \%$ neutral buffered formalin at $2.4 \mathrm{kPa}$ for $3 \mathrm{~h}$. The lungs were removed and then fixed additionally in $10 \%$ neutral buffered formalin for $72 \mathrm{~h}$.

Five-micrometer-thick sections from the paraffin block of four groups of P10 and P21 old pups' lungs were cut and stained with hematoxylin and eosin for tissue morphology. Six random nonoverlapping fields per pup in the distal lung sections were used for the morphometric examinations. Sections were photographed using a digital camera. Photographs were analyzed using Image J (NIH) RAC was obtained as described by Emery and Mithal (20). AWT was calculated using $\mathrm{AWT}=\mathrm{VD}_{\mathrm{T}}$ (tissue volume density) $\times \mathrm{L}_{\mathrm{m}}$ (cord length) (ref. 21). For the measurement of secondary septa, elastin was stained with resorcin-fuchsin and Van Gieson's solution. To quantify capillary density, we measured the rat endothelial cell antigen-1-immunostained area compared with total parenchymal cell area, using Colour Deconvolution (22). 


\section{Caffeine in hyperoxic lung injury $\mid$ Articles}
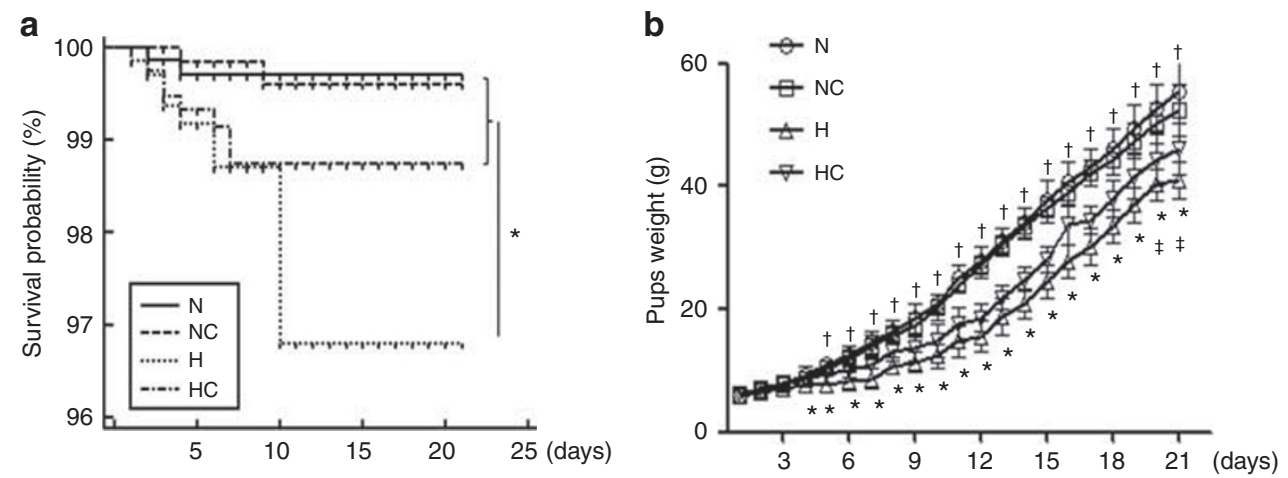

Figure 2. Hyperoxia decreased the survival and weight gain in pups, and early caffeine treatment ameliorated the growth-inhibiting effect of hyperoxia. (a) The survival rate decreased in pups exposed to hyperoxia, and daily caffeine treatment improved the survival rate ${ }^{*} P<0.05, n \geq 50$ per group). Although there was a significant difference, $>95 \%$ of all rat pups survived the experiments. (b) Weight gain was significantly reduced by hyperoxia from the fourth day (P4) after birth. Pups in the hyperoxia group were on average $26 \%$ lighter than the ones from the normoxia group at $\mathrm{P} 21$. Caffeine attenuated the weight loss effect in the hyperoxia group but caused weight loss in the normoxia group. Normoxia ( $\mathrm{N}, \mathrm{O}$ ), normoxic pups treated with $20 \mathrm{mg} / \mathrm{kg}$ caffeine per day $(\mathrm{NC}, \square)$, age-matched hyperoxia controls $(\mathrm{H}, \Delta)$, and hyperoxia pups treated with $20 \mathrm{mg} / \mathrm{kg}$ caffeine per day $(\mathrm{HC}, \nabla)$. Significant restriction of the pups' weight gain by hyperoxia was seen since $\mathrm{P} 4\left({ }^{*} P<0.05, n \geq 50\right.$ per group). From $\mathrm{P} 5$, caffeinetreated pups' weight was significantly higher compared with that of saline-treated pups in hyperoxia $\left({ }^{\dagger} P<0.05, n \geq 34\right.$ per group). Body weights of the caffeine-treated normoxia group were lower than those of the saline-treated group at P21 $\left({ }^{\ddagger} P<0.01, n=17\right.$ per group). $\mathrm{H}$, hyperoxia; $\mathrm{HC}$, hyperoxia+caffeine; N, normoxia; NC, normoxia+caffeine.

\section{GCH1 Promoter Methylation}

DNA extracted from $\sim 5 \mathrm{mg}$ of lung tissue was purified using the PureLink Genomic DNA Mini kit. Then, $500 \mathrm{ng}$ of extracted genomic DNA was bisulfate-converted using the EZ DNA Methylation kit. The pyrosequencing system (PyroMark Q96 MD, Qiagen) was used to quantify methylated CpGs. Primer sequences for the GCH1 promoter region were designed with PyroMark Q96 MD.

Forward primer: 5'-GGTTTTTTAGTTGGAGGTTTGTTTTAGA TT-3'

Reverse primer: 5' -biotin-ACCCATTAATACACCTTACACC-3'

Sequencing primer: 5'-AATTTTTGTTTAGGTGATAGTTATA-3'.

\section{Immunoprecipitation}

Immunoprecipitation was performed to detect ubiquitinated GCH1. Lung tissue homogenates were obtained as we described. An amount of $1 \mathrm{mg}$ of each lysate protein was incubated overnight at $4{ }^{\circ} \mathrm{C}$ with anti-GCH1. Immunocomplexes were adsorbed to $100 \mu \mathrm{l}$ of protein A/G plus agarose beads (Thermo Fisher Scientific, Waltham, MA). Samples were denatured with SDS loading buffer, and proteins were separated by SDS-polyacrylamide gel electrophoresis. Western blotting was performed with anti-ubiquitin and anti-GCH1 antibodies. The ratio of ubiquitin/total GCH1 was assessed.

\section{Statistical Analysis}

Statistical analysis was performed using GraphPad Prism version 5 for Windows (GraphPad Software, San Diego, CA). Values are expressed as mean $\pm S D$. Significant differences among groups were determined by analysis of variance with Student-Newman-Keuls post hoc test. A $P$ value $<0.05$ was considered statistically significant.

\section{RESULTS}

\section{Caffeine Decreases Mortality and Improves Weight Gain in Hyperoxia-Exposed Rat Pups}

A total of 20 pregnant dams (4 dams per experiment) were used in this study. The mortality rate increased in rat pups exposed to hyperoxia compared with that in the pups exposed to normoxia; however, daily caffeine treatment decreased the mortality rate in the hyperoxia group (Figure 2a). Pups' weight (Figure 2b) was significantly decreased by hyperoxia as early as P4. Daily caffeine therapy improved postnatal weight gain. Conversely, caffeine reduced the weight gain after P20 in the normoxia group.

\section{Serum Caffeine Levels}

Serum caffeine levels were undetectable in the saline-treated pups. The levels for the caffeine-treated pups were $19.3 \pm 5.3 \mu \mathrm{g} / \mathrm{ml}(n=12)$ at P10 and $18.4 \pm 4.9 \mu \mathrm{g} / \mathrm{ml}(n=12)$ at P21 without difference between groups (hyperoxia+caffeine vs. normoxia+caffeine, $P=0.8$ by analysis of variance).

\section{Effects of Hyperoxia and Caffeine on Lung Structure}

There were no differences in the radial alveolar count (RAC), alveolar wall thickness (AWT; Figure 3a), secondary septa formation (Figure 3b), and capillary count (Figure 3c) between caffeine-treated and saline-treated normoxia groups. Hyperoxia exposure impaired lung growth at P10 with decreased RAC (19\%), increased AWT (60\%), and a marked decrease in the number of secondary septa (40\%) and capillaries (60\%). Caffeine treatment improved alveolar development during hyperoxia by increasing RAC (17\%), decreasing AWT (30\%), increasing the number of secondary septa (16\%), and increasing capillary density (100\%) compared with the saline-treated group.

Continuation of caffeine treatment during recovery in room air maintained the beneficial effect on lung structure in the hyperoxia group (Figure 2). At P21, the lung structure remained impaired in the hyperoxia+saline group with decreased RAC (37\%), increased wall thickness (60\%), and decreased number of secondary septa (28\%) and capillaries (54\%). The lung structure remained significantly improved by caffeine in the hyperoxia group at P21.

\section{Effects of Caffeine on cAMP Level}

Exposure to hyperoxia decreased the cAMP levels in the lungs compared with the normoxia controls. Caffeine treatment 


\section{Articles | jing et al.}

a

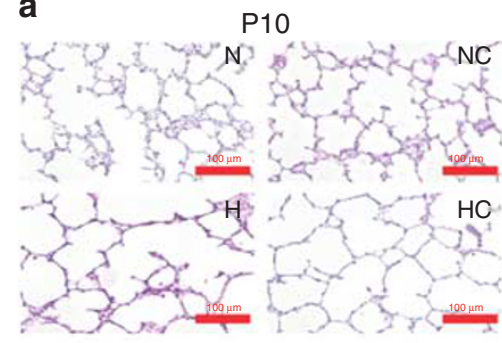

b

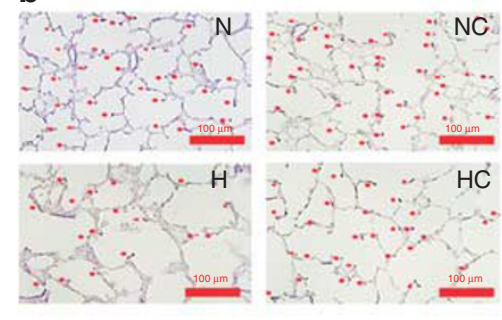

C

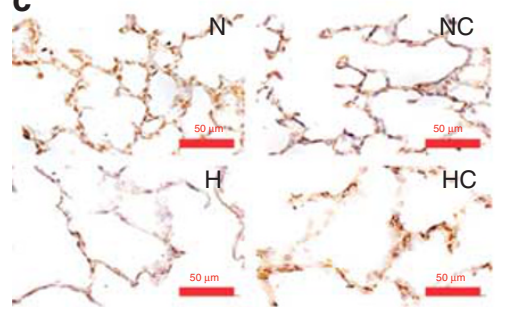

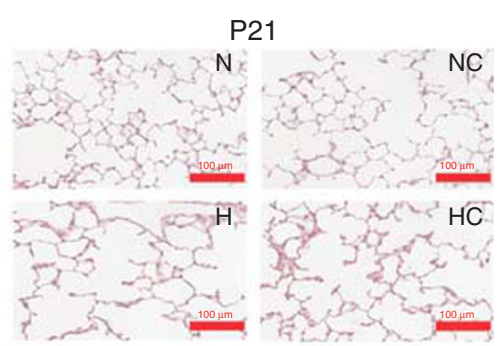
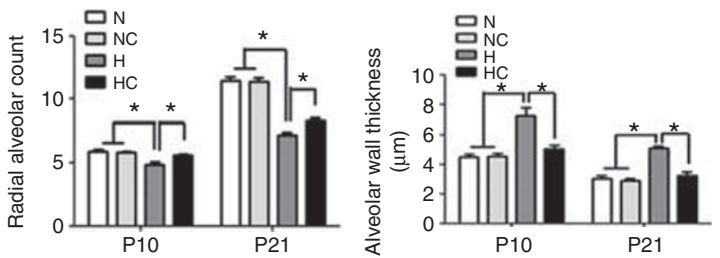
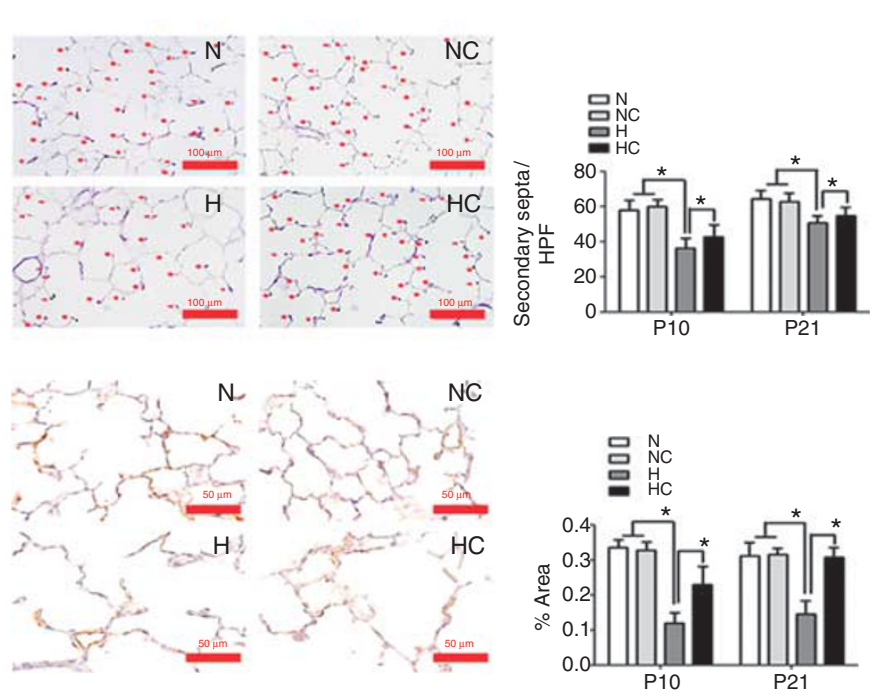

Figure 3. Caffeine ameliorates the effects of hyperoxia on lung growth. Lung sections were stained with hematoxylin and eosin for histology at P10 and P21 (a). The radial alveolar count (RAC) was decreased by hyperoxia. Caffeine increased the RAC in the hyperoxia group. Alveolar wall thickness (AWT) was increased in hyperoxia. Caffeine decreased the AWT in the hyperoxia group (a). Weigert's resorcin-fuchsin stain for elastin (purple dots) showed decreased secondary septa formation in hyperoxia at P10 and P21 (b). The area of rat endothelial cell antigen-1 (RECA-1)-immunostained endothelial cells as a proportion of total lung parenchymal cell area was measured using Image $\mathrm{J}$. Ten random fields were chosen from each slide for seven to eight animals at each time point $(\mathbf{c})$. Caffeine-treated pups maintained their blood vessel counts in hyperoxia. ${ }^{*} P<0.01, n(\mathrm{~N})=8, n(\mathrm{NC})=8$, $n(\mathrm{H})=7, n(\mathrm{HC})=7$.

restored the cAMP levels in the hyperoxia-exposed lungs at P10. At P21, cAMP levels of the hyperoxia group were lower than those of the normoxia group, with no difference between caffeine- and saline-treated hyperoxic pups (Figure 4).

Effects of Caffeine on BH4 Metabolism and Angiogenic Factors To investigate the changes in angiogenesis by hyperoxia, we evaluated the proteins that are known to modulate angiogenesis, including vascular endothelial cell growth factor (VEGF) and eNOS, and proteins that are known to affect $\mathrm{BH} 4$ levels, including GCH1 and dihydrofolate reductase (DHFR). Hyperoxia decreased VEGF and phospho-eNOS serine ${ }^{1177}$ (p-eNOS ${ }^{1177}$ ) levels at P10, and caffeine partially abrogated this effect (Figure 5a). Recovery in room air restored the VEGF expression in the hyperoxia group to normoxic levels at $\mathrm{P} 21$, but p-eNOS ${ }^{117}$ levels remained lower in the hyperoxia group compared with those in the normoxia group; caffeine significantly increased $\mathrm{p}$-eNOS levels in the hyperoxia group at P21 compared with those in the saline-treated group (Figure 5a).

Hyperoxia also decreased GCH1 and phospho-serine ${ }^{51}$ GCH1 (p-GCH1 ${ }^{51}$ ) (17) levels in the lungs at P10; caffeine treatment significantly increased both $\mathrm{GCH} 1$ and $\mathrm{p}-\mathrm{GCH} 1^{51}$ levels in the hyperoxia group. The GCH1 levels show a persistent decrease at P21 after hyperoxia; caffeine increased the GCH1 levels at P21 in the hyperoxia group (Figure $5 \mathbf{b}$ ). The $\mathrm{p}-\mathrm{GCH}{ }^{51}$ levels, however, recovered to normoxic levels by P21, with no additional changes with caffeine treatment (Figure 5b). DHFR, the enzyme for salvage pathway BH4 formation, levels were unchanged by either hyperoxia or caffeine treatment. Changes in BH4 levels paralleled the changes in GCH1 expression; BH4 levels decreased in hyperoxia alone at P10 and P21, and were increased at both time points by caffeine (Figure $5 \mathrm{c}$ ).

\section{Methylation of the GCH1 Promoter and Ubiquitination of GCH1} Previous studies demonstrated that hyperoxia-induced lung injury is associated with alterations in the methylation of promoter regions of certain genes $(23,24)$. The GCH1 promoter area contains 12 potential methylation sites. We did not observe difference in methylation levels of GCH1 promoter among the four groups (Figure 6a). The methylation percent was very low in all groups, suggesting 
that $G C H 1$ gene has a high transcriptional potential in the postnatal developing lungs.

Immunoprecipitation analysis demonstrated an increase in GCH1 ubiquitination by hyperoxia in the lungs at both P10

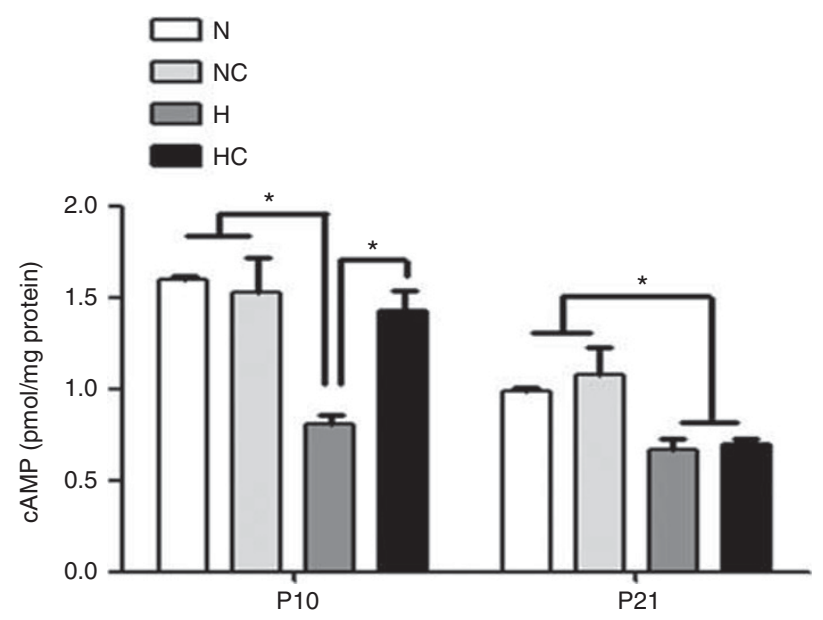

Figure 4. Levels of cyclic AMP (CAMP) decreased at P10 and P21 in hyperoxia; caffeine restored CAMP to normoxia levels at P10. Caffeine did not increase cAMP levels at P21 in the hyperoxia group. ${ }^{*} P<0.05$, $n=6$ per group. and P21 (Figure 6b), when compared with normoxia in the lungs. Caffeine decreased GCH1 ubiquitination at both P10 and $\mathrm{P} 21$.

\section{DISCUSSION}

BPD is a common cause of long-term morbidity, with $>10,000$ new cases annually in the United States, increasing the healthcare resource utilization for premature babies. Impaired angiogenesis is believed to be a key contributor to decreased alveolar growth in BPD $(3,25)$ and may further increase the risk of pulmonary hypertension. Our study investigated the mechanism by which caffeine improves lung growth during hyperoxia. We observed that caffeine helps maintain lung growth under hyperoxia, in part, by ameliorating impaired angiogenesis through improving GCH1 activity.

Premature infants commonly receive oxygen and mechanical ventilation right after birth for their respiratory support. Both oxygen and mechanical ventilation increase oxidative stress in the immature lungs (26-28). Increased oxidative stress depletes BH4 in vascular endothelial cells (10). BH4 promotes eNOS association with heat-shock protein 90 and eNOS dimerization, both of which are required steps for adequate eNOS coupling (17). BH4 is also an intracellular antioxidant (29) that may facilitate NO bioavailability and
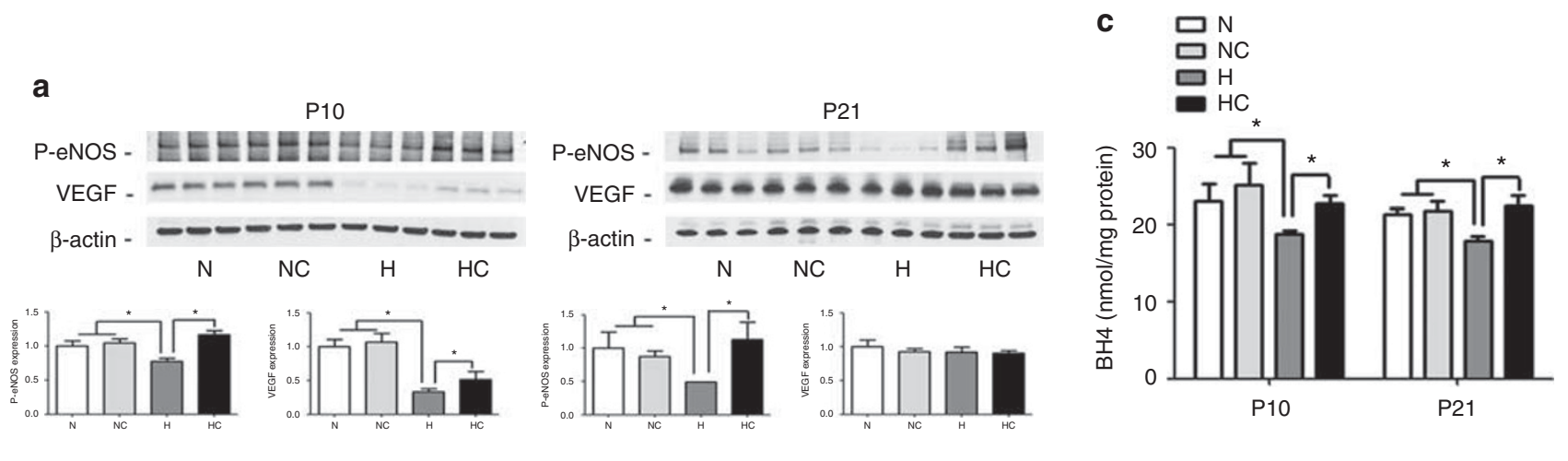

b
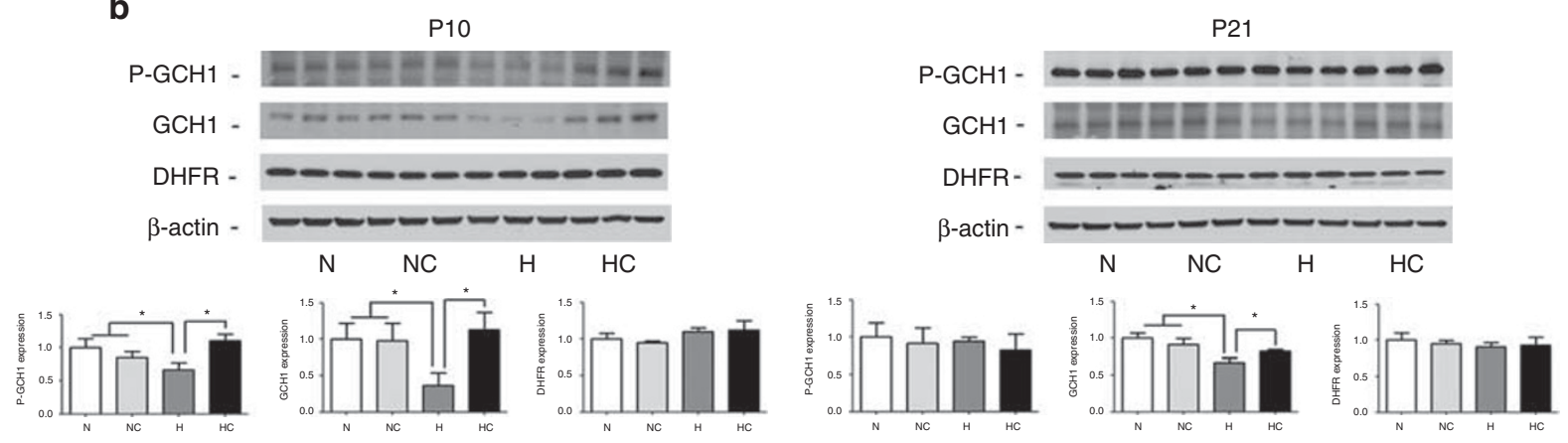

Figure 5. Effects of hyperoxia and caffeine on the levels of angiogenesis proteins and BH4 in the rat lungs. (a) Hyperoxia decreased vascular endothelial cell growth factor (VEGF) levels at P10, but not at P21; caffeine restored VEGF levels in the hyperoxia group at P10. Hyperoxia decreased endothelial nitric oxide synthase serine ${ }^{1177}$ phosphorylation (p-eNOS ${ }^{1177}$ ) at both P10 and P21, and caffeine increased p-eNOS levels at both time points. (b) Hyperoxia decreased phospho-GTP cyclohydrolase 1 serine ${ }^{51}$ (p-GCH1 ${ }^{51}$ ) and GTP cyclohydrolase 1 (GCH1) at P10, and caffeine increased their levels. There was no change in $\mathrm{p}-\mathrm{GCH} 1$ at P21, whereas $\mathrm{GCH} 1$ levels were lower in the hyperoxia group and this was increased by caffeine. There was no change in dihydrofolate reductase (DHFR) levels in any group at either P10 or P21. (c) The changes in tetrahydrobiopterin (BH4) levels paralleled the changes in $\mathrm{GCH} 1$ level, with the hyperoxia group showing decreases at both P10 and P21. Caffeine increased BH4 levels during hyperoxia at P10 and P21. ${ }^{*} P<0.05, n=6$ per group. 
a

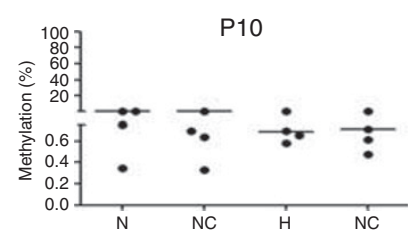

b

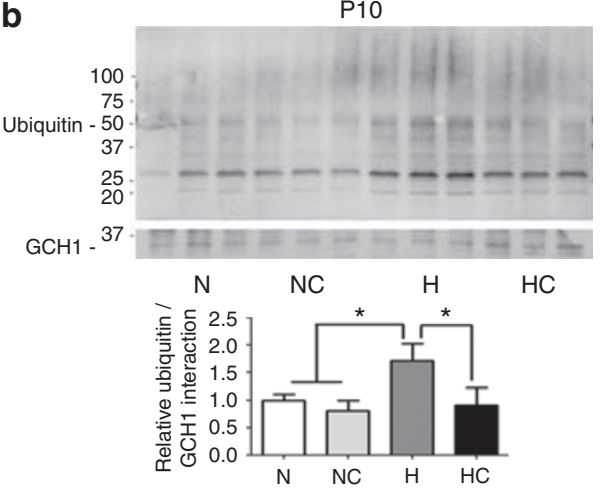

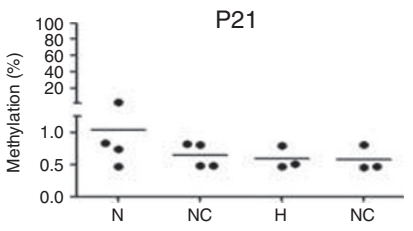
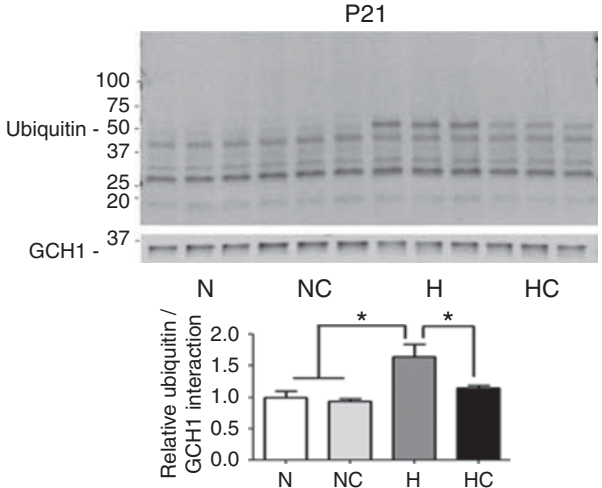

Figure 6. (a) $\mathrm{GCH} 1$ promoter methylation levels were not affected by either hyperoxia or caffeine at both P10 and P21. (b) Hyperoxia and caffeine effects on $\mathrm{GCH} 1$ levels are mainly at the post-translational level. Hyperoxia increased GCH1 ubiquitination and caffeine decreased this effect at both $\mathrm{P} 10$ and P21. ${ }^{*} \mathrm{P}>0.05, n=4$ for P10 all four groups; $n=4$ for $\mathrm{N}$ and NC at P21, but $n=3$ for $\mathrm{H}$ and HC at P21.

stabilize soluble guanylate cyclase (30). Studies in animal models have demonstrated that $\mathrm{NO}$ is both the activator and mediator of VEGF, and its effects on angiogenesis and alveolar growth in the developing lungs is well described $(31,32)$. We previously reported that increased oxidative stress depletes BH4 in pulmonary artery endothelial cells, which contributes to the impaired angiogenesis in the developing lungs (33).

In this study, we showed that DHFR, which regenerates BH4 through the salvage pathway (34), did not change with either hyperoxia or caffeine treatment. On the other hand, we observed that GCH1 and p-GCH1 ${ }^{51}$ expression in the pups' lungs was significantly reduced by hyperoxia exposure and restored by caffeine treatment, indicating that the changes in BH4 levels were mainly due to the changes in GCH1 levels and the post-translational modifications.

The mechanism(s) by which hyperoxia decreases GCH1 levels remain unclear. Our data indicate that neither hyperoxia nor caffeine treatment alter the methylation level of the GCH1 gene promoter area. Some previous studies identified that oxidative stress stimulates ubiquitin-proteasomal protein degradation $(35,36)$. We investigated whether ubiquitin-proteasomal degradation explains the changes we observed in GCH1 protein levels in hyperoxia-exposed rat lungs. Our results suggested that hyperoxia increased GCH1 ubiquitination in the lungs; this effect was reversed by caffeine treatment. The increase in cAMP levels we observed with caffeine treatment may provide the protection to $\mathrm{GCH} 1$ by reducing both ubiquitin-dependent degradation and reduction in reactive oxygen species formation (37).

Although we show that early caffeine treatment ameliorates hyperoxia-induced lung injury, our results are different from those reported by Londhe and colleagues (38) Londhe showed that caffeine aggravates the impaired growth of hyperoxiaexposed lungs with increased apoptosis in alveolar cells. These contradicting observations deserve further investigation, but there are at least two distinct differences between the two studies. Our study used a higher dose of caffeine $(20 \mathrm{mg} / \mathrm{kg}$ per day) than that reported by Londhe and colleagues $(10 \mathrm{mg} / \mathrm{kg}$ per day). However, the measured serum caffeine levels in our study were within the clinically suggested therapeutic range $(5-20 \mu \mathrm{g} / \mathrm{ml})$ (39). Whether a higher caffeine dose confers the observed benefits deserves future investigation. The studies by Londhe used inbred mouse pups, whereas we used outbred Sprague-Dawley rat pups, and it is possible to have an inter-strain difference in response. Future studies in different models of lung injury are necessary to validate the potential beneficial effects of caffeine.

We observed that caffeine treatment provides benefits only in hyperoxia-exposed pups. Hyperoxia decreased weight gain in our study even with switching of the nursing dams, which may be secondary to our $>90 \%$ oxygen environment, but caffeine improved weight gain despite exposure to hyperoxia. In fact, caffeine decreased postnatal weight gain in the normoxia group. However, most extremely premature infants require supplemental oxygen to provide adequate oxygenation early in life, so the early caffeine treatment in this susceptible group with poor weight gain would be less concerning.

In conclusion, our study provided plausible biological mechanisms by which early caffeine treatment protects the developing lungs from hyperoxia-induced injury. By maintaining adequate GCH1 activity, caffeine can preserve eNOS function during hyperoxia exposure.

\section{ACKNOWLEDGMENTS}

We acknowledge Dr Jianhai Du for the protocol for BH4 measurement. 


\section{Caffeine in hyperoxic lung injury $\quad$ Articles}

\section{STATEMENT OF FINANCIAL SUPPORT}

The study was supported by National Institutes of Health Grants HL-080468S (YS); R01 HL057268, Muma Endowed Chair and Children's Research Institute (GGK); R03HD-073274, UL1TR000055 and UL1TR001436 Advancing Healthier Wisconsin Foundation (R-JT).

Disclosure: The authors declare no conflict of interest.

\section{REFERENCES}

1. Yoon BH, Romero R, Jun JK, et al. Amniotic fluid cytokines (interleukin6 , tumor necrosis factor-alpha, interleukin-1 beta, and interleukin-8) and the risk for the development of bronchopulmonary dysplasia. Am J Obstet Gynecol 1997;177:825-30.

2. Saugstad OD. Oxygen and oxidative stress in bronchopulmonary dysplasia. J Perinat Med 2010;38:571-7.

3. Abman SH. Bronchopulmonary dysplasia: "a vascular hypothesis". Am J Respir Crit Care Med 2001;164:1755-6.

4. Brueckl C, Kaestle S, Kerem A, et al. Hyperoxia-induced reactive oxygen species formation in pulmonary capillary endothelial cells in situ. Am J Respir Cell Mol Biol 2006;34:453-63.

5. Waters CM. Reactive oxygen species in mechanotransduction. Am J Physiol Lung Cell Mol Physiol 2004;287:L484-5.

6. Cooke JP, Losordo DW. Nitric oxide and angiogenesis. Circulation 2002;105:2133-5.

7. Marletta MA. Nitric oxide synthase structure and mechanism. J Biol Chem 1993;268:12231-4.

8. Moens AL, Kass DA. Tetrahydrobiopterin and cardiovascular disease. Arterioscler Thromb Vasc Biol 2006;26:2439-44.

9. Vaporidi K, Francis RC, Bloch KD, Zapol WM. Nitric oxide synthase 3 contributes to ventilator-induced lung injury. Am J Physiol Lung Cell Mol Physiol 2010;299:L150-9.

10. Burri PH. Structural aspects of postnatal lung development-alveolar formation and growth. Biol Neonate 2006;89:313-22.

11. O'Reilly M, Thébaud B. Animal models of bronchopulmonary dysplasia II: the term rat models. Am J Physiol Lung Cell Mol Physiol 2014;307:L948-58.

12. Aranda J, Beharry K, Valencia GB, Natarajan G, Davis J. Caffeine and impact on neonatal morbidities. J Maternal-Fetal Neonat Med 2010;23: 20-3.

13. Kassim Z, Greenough A, Rafferty GF. Effect of caffeine on respiratory muscle strength and lung function in prematurely born, ventilated infants. Eur J Pediatr 2009;168:1491-5.

14. Schmidt B, Roberts RS, Davis P, et al. Caffeine therapy for apnea of prematurity. N Engl J Med 2006;354:2112-21.

15. Lodha A, Seshia M, McMillian DD, et al. Association of early caffeine administration and neonatal outcomes in very preterm neonates. JAMA Pediatr 2015;169:33-8.

16. Kumar S, Sun X, Sharma S, et al. GTP cyclohydrolase I expression is regulated by nitric oxide: role of cyclic AMP. Am J Physiol Lung Cell Mol Physiol 2009;297:L309-17.

17. Du J, Wei N, Xu H, et al. Identification and functional characterization of phosphorylation sites on GTP cyclohydrolase I. Arterioscler Thromb Vasc Biol. 2009;29:2161-8.

18. Teng RJ, Du J, Xu H, et al. Sepiapterin improves angiogenesis of pulmonary artery endothelial cells in persistent pulmonary hypertension of the newborn by recoupling endothelial nitric oxide synthase. Am J Physiol Lung Cell Mol Physiol 2011;301:L334-45.

19. Du J, Teng RJ, Lawrence $M$, et al. The protein partners of GTP cyclohydrolase I in rat organs. PLoS ONE 2012;7:e33991.

20. Emery JL, Mithal A. The number of alveoli in the terminal respiratory unit of man during late intrauterine life and childhood. Arch Dis Child 1960;35:544-7.
21. Choi CW, Kim BI, Hong JS, Kim EK, Kim HS, Choi JH. Bronchopulmonary dysplasia in a rat model induced by intra-amniotic inflammation and postnatal hyperoxia: morphometric aspects. Pediatr Res 2009;65:323-7.

22. Ruifrok AC, Johnston DA. Quantification of histochemical staining by color deconvolution. Anal Quant Cytol Histol 2001;23:291-9.

23. Yue X, Fu J, Xue X, et al. Detection of p16 promoter methylation in premature rats with chronic lung disease induced by hyperoxia. Pediatr Int 2010;52:520-6.

24. Lagishetty V, Parthasarathy PT, Phillips O, et al. Dysregulation of CLOCK gene expression in hyperoxia-induced lung injury. Am J Physiol Cell Physiol 2014;306:C999-1007.

25. Ladha F, Bonnet S, Eaton F, Hashimoto K, Korbutt G, Thébaud B. Sildenafil improves alveolar growth and pulmonary hypertension in hyperoxia-induced lung injury. Am J Respir Crit Care Med 2005;172: 750-6.

26. Kwak DJ, Kwak SD, Gauda EB. The effect of hyperoxia on reactive oxygen species (ROS) in rat petrosal ganglion neurons during development using organotypic slices. Pediatr Res 2006;60:371-6.

27. Schock BC, Sweet DG, Halliday HL, Young IS, Ennis M. Oxidative stress in lavage fluid of preterm infants at risk of chronic lung disease. Am J Physiol Lung Cell Mol Physiol 2001;281:L1386-91.

28. Gitto E, Pellegrino S, D'Arrigo S, Barberi I, Reiter RJ. Oxidative stress in resuscitation and in ventilation of newborns. Eur Respir J 2009;34:1461-9.

29. Kojima S, Ona S, Iizuka I, Arai T, Mori H, Kubota K. Antioxidative activity of 5,6,7,8-tetrahydrobiopterin and its inhibitory effect on paraquat-induced cell toxicity in cultured rat hepatocytes. Free Radic Res 1995;23:419-30.

30. Schmidt K, Neubauer A, Kolesnik B, et al. Tetrahydrobiopterin protects soluble guanylate cyclase against oxidative inactivation. Mol Pharmacol 2012;82:420-7.

31. Lin YJ, Markham NE, Balasubramaniam V, et al. Inhaled nitric oxide enhances distal lung growth after exposure to hyperoxia in neonatal rats. Pediatr Res 2005;58:22-9.

32. Balasubramaniam V, Tang JR, Maxey A, Plopper CG, Abman SH. Mild hypoxia impairs alveolarization in the endothelial nitric oxide synthasedeficient mouse. Am J Physiol Lung Cell Mol Physiol 2003;284:L964-71.

33. Teng RJ, Eis A, Bakhutashvili I, Arul N, Konduri GG. Increased superoxide production contributes to the impaired angiogenesis of fetal pulmonary arteries with in utero pulmonary hypertension during transitional stage. Am J Physiol Lung Cell Mol Physiol 2009;297: L184-95.

34. Crabtree MJ, Tatham AL, Hale AB, Alp NJ, Channon KM. Critical role for tetrahydrobiopterin recycling by dihydrofolate reductase in regulation of endothelial nitric-oxide synthase coupling: relative importance of the de novo biopterin synthesis versus salvage pathways. J Biol Chem 2009;284:28128-36.

35. Medicherla B, Goldberg AL. Heat shock and oxygen radicals stimulate ubiquitin-dependent degradation mainly of newly synthesized proteins. J Cell Biol 2008;182:663-73.

36. Sharma S, Sun X, Kumar S, et al. Preserving mitochondrial function prevents the proteasomal degradation of GTP cyclohydrolase I. Free Radic Biol Med 2012;53:216-9.

37. Huang H, Wang H, Figueiredo-Pereira ME. Regulating the ubiquitin/ proteasome pathway via cAMP-signaling: neuroprotective potential. Cell Biochem Biophys 2013;67:55-66.

38. Dayanim S, Lopez B, Maisonet TM, Grewal S, Londhe VA. Caffeine induces alveolar apoptosis in the hyperoxia-exposed developing mouse lung. Pediatr Res 2014;75:395-402.

39. Gal P. Caffeine therapeutic drug monitoring is necessary and cost-effective. J Pediatr Pharmacol Ther 2007;12:212-5. 